\title{
RESOLVING THE CHEMISTRY IN THE DISK OF TW HYDRAE. I. DEUTERATED SPECIES

\author{
Chunhua Qi, ${ }^{1}$ David J. Wilner, ${ }^{1}$ Yuri Aikawa, ${ }^{2}$ Geoffrey A. Blake, ${ }^{3}$ and Michiel R. Hogerheijde ${ }^{4}$
} \\ Received 2007 October 5; accepted 2008 March 18
}

\begin{abstract}
We present Submillimeter Array observations of several deuterated species in the disk around the classical T Tauri star TW Hydrae at arcsecond scales, including detections of the DCN $J=3-2$ and $\mathrm{DCO}^{+} J=3-2$ lines and upper limits to the $\mathrm{HDO} 3_{1,2}-2_{2,1}$, ortho- $\mathrm{H}_{2} \mathrm{D}^{+} 1_{1,0}-1_{1,1}$, and para- $\mathrm{D}_{2} \mathrm{H}^{+} 1_{1,0}-1_{0,1}$ transitions. We also present observations of the $\mathrm{HCN} J=3-2, \mathrm{HCO}^{+} J=3-2$, and $\mathrm{H}^{13} \mathrm{CO}^{+} J=4-3$ lines for comparison with their deuterated isotopologues. We constrain the radial and vertical distributions of various species in the disk by fitting the data using a model where the molecular emission from an irradiated accretion disk is sampled with a two-dimensional Monte Carlo radiative transfer code. We find that the distribution of $\mathrm{DCO}^{+}$differs markedly from that of $\mathrm{HCO}^{+}$. The $\mathrm{D} / \mathrm{H}$ ratios inferred change by at least 1 order of magnitude $(0.01-0.1)$ for radii $<30$ to $\geq 70 \mathrm{AU}$, and there is a rapid falloff of the abundance of $\mathrm{DCO}^{+}$at radii larger than $90 \mathrm{AU}$. Using a simple analytical chemical model, we constrain the degree of ionization, $x(e-)=n(e-) / n\left(\mathrm{H}_{2}\right)$, to be $\sim 10^{-7}$ in the disk layer(s) where these molecules are present. Provided the distribution of $\mathrm{DCN}$ follows that of $\mathrm{HCN}$, the ratio of $\mathrm{DCN}$ to $\mathrm{HCN}$ is determined to be $(1.7 \pm 0.5) \times 10^{-2}$; however, this ratio is very sensitive to the poorly constrained vertical distribution of $\mathrm{HCN}$. The resolved radial distribution of $\mathrm{DCO}^{+}$indicates that in situ deuterium fractionation remains active within the TW Hydrae disk and must be considered in the molecular evolution of circumstellar accretion disks.
\end{abstract}

Subject headings: circumstellar matter - comets: general — ISM: molecules — planetary systems: protoplanetary disks - stars: individual (TW Hydrae) — stars: pre-main-sequence

Online material: color figures

\section{INTRODUCTION}

Millimeter-wave interferometers have imaged the gas and dust surrounding over a dozen T Tauri and Herbig Ae stars (see Dutrey et al. 2007 for a review). These studies demonstrate the potential to dramatically improve our understanding of disk physical and chemical structure, providing insights that will ultimately enable a more comprehensive understanding of star and planet formation. As analogs to the solar nebula, these circumstellar disks offer a unique opportunity to study the conditions during the planet formation process, especially the complex chemical evolution that must occur. In the outer parts of the disk directly accessible to millimeter-wave interferometry, observations of deuterated species are particularly important because they can constrain the origin of primitive solar system bodies such as comets and other icy planetesimals.

Deuterated molecule chemistry is sensitive to the temperature history of interstellar and circumstellar gas, as well as to densitysensitive processes such as molecular depletion in the cold $(<20 \mathrm{~K})$ and dense $\left(>10^{6} \mathrm{~cm}^{-3}\right)$ disk midplane. The similarity of molecular $\mathrm{D} / \mathrm{H}$ ratios between comets and high-mass hot cores has been used to argue for an "interstellar" origin of cometary matter, but ambiguity remains, and this argument is not secure (Bergin et al. 2007). Aikawa \& Herbst (1999) investigate the chemistry of deuterium-bearing molecules in the outer regions of protoplanetary disks and find that molecules formed in the

\footnotetext{
1 Harvard-Smithsonian Center for Astrophysics, 60 Garden Street, MS 42, Cambridge, MA 02138; cqi@cfa.harvard.edu,dwilner@cfa.harvard.edu.

2 Department of Earth and Planetary Sciences, Kobe University, Kobe 6578501, Japan; aikawa@kobe-u.ac.jp.

3 Divisions of Geological and Planetary Sciences and Chemistry and Chemical Engineering, California Institute of Technology, MS 150-21, Pasadena, CA 91125; gab@gps.caltech.edu.

4 Leiden Observatory, Leiden University, P.O. Box 9513, 2300 RA Leiden, Netherlands; michiel@strw.leidenuniv.nl.
}

disk have $\mathrm{D} / \mathrm{H}$ ratios similar to those in comets. To date, the determination of $\mathrm{D} / \mathrm{H}$ ratios in disks has been limited to the measurements of $\mathrm{DCO}^{+} / \mathrm{HCO}^{+}$in two classical T Tauri stars (CTTSs) with single-dish telescopes (TW Hydrae, van Dishoeck et al. 2003; DM Tauri, Guilloteau et al. 2006), but $\mathrm{DCO}^{+}$has not been observed in comets. Although $\mathrm{H}_{2} \mathrm{D}^{+}$and HDO lines have been detected in disks (Ceccarelli et al. 2004, 2005), there are no corresponding $\mathrm{H}_{3}^{+}$and $\mathrm{H}_{2} \mathrm{O}$ observations that allow derivations of the $\mathrm{D} / \mathrm{H}$ ratio. Therefore, direct measurements in disks of species observed in comets, such as DCN and $\mathrm{HCN}$, will be important to help relate deuterium fractionation in disks to that in comets.

Deuterated molecules in cold prestellar and protostellar cores are found to be enhanced by orders of magnitude over the elemental $\mathrm{D} / \mathrm{H}$ abundance ratio of $1.5 \times 10^{-5}$ through fractionation in the gas phase at low temperatures, an effect driven primarily by deuterated $\mathrm{H}_{3}^{+}$, which is formed by an exchange reaction between $\mathrm{H}_{3}^{+}$and $\mathrm{HD}$, then transfers its deuteron to neutral species. Theoretical models of disks (Aikawa et al. 2002; Willacy 2007) with realistic temperature and density structure show that the $\mathrm{DCO}^{+}$/ $\mathrm{HCO}^{+}$ratio increases with radius due to the decreasing temperature moving out from the star. Spatially resolved data of deuterated molecules will help test disk physical models through these chemical consequences.

The current capabilities of millimeter-wave observatories are limited both by sensitivity and by the small angular size of circumstellar disks, which make spatially resolving the deuterium fractionation difficult. In this work we take advantage of the proximity of the most nearby CTTS, TW Hydrae, which is surrounded by a disk of radius $3.5^{\prime \prime}$, or $200 \mathrm{AU}$, at a distance of $56 \mathrm{pc}$ (Qi et al. 2004 ), and a possible orbiting planet of mass $9.8 \pm 3.3 M_{\mathrm{Jup}}$ at 0.04 AU (Setiawan et al. 2008), to study the physical and chemical structure of a protoplanetary environment at high spatial and spectral resolution using interferometry. The TW Hydrae disk is viewed nearly face-on and so is well positioned to investigate the radial distribution of molecular emission. Although the current 
TABLE 1

Observational Parameters for SMa TW Hydrae (Detected Species)

\begin{tabular}{|c|c|c|c|c|c|}
\hline Parameter & $\mathrm{HCN} 3-2$ & $\mathrm{HCO}^{+} 3-2$ & $\mathrm{H}^{13} \mathrm{CO}^{+} 4-3$ & DCN $3-2^{a}$ & $\mathrm{DCO}^{+} 3-2$ \\
\hline Rest frequency $(\mathrm{GHz}) \ldots \ldots \ldots \ldots \ldots \ldots \ldots$ & 265.88618 & 267.55762 & 346.99854 & 217.23863 & 216.11260 \\
\hline \multirow[t]{3}{*}{ 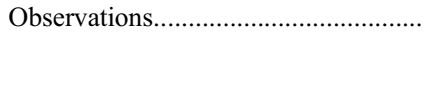 } & 2005 Mar 4 & 2005 Mar 4 & 2006 Dec 28 & 2006 Apr 28 & 2006 Apr 28 \\
\hline & 2005 Apr 21 & 2005 Apr 21 & $\ldots$ & $\ldots$ & 2006 Feb 3 \\
\hline & 2005 Apr 26 & 2005 Apr 26 & $\ldots$ & $\ldots$ & $\ldots$ \\
\hline Antennas used.. & 7 & 7 & 8 & 8 & 8 \\
\hline \multirow[t]{2}{*}{ Synthesized beam ......................... } & $1.6^{\prime \prime} \times 1.1^{\prime \prime}$ & $1.6^{\prime \prime} \times 1.1^{\prime \prime}$ & $4.1^{\prime \prime} \times 1.8^{\prime \prime}$ & $5.9^{\prime \prime} \times 3.2^{\prime \prime}$ & $2.6^{\prime \prime} \times 1.6^{\prime \prime}$ \\
\hline & P.A. $=-0.5^{\circ}$ & P.A. $=-6.3^{\circ}$ & P.A. $=3.3^{\circ}$ & P.A. $=-1.5^{\circ}$ & P.A. $=2.8^{\circ}$ \\
\hline Channel spacing $\left(\mathrm{km} \mathrm{s}^{-1}\right) \ldots \ldots \ldots \ldots \ldots$ & 0.23 & 0.23 & 0.70 & 0.56 & 0.28 \\
\hline 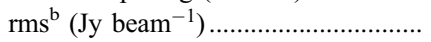 & 0.35 & 0.29 & 0.16 & 0.10 & 0.10 \\
\hline 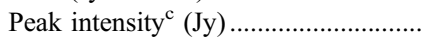 & 4.7 & 2.0 & 0.70 & 0.31 & 0.56 \\
\hline
\end{tabular}

a Only compact configuration data used for DCN observation.

b SNR limited by the dynamic range.

c Intensity averaged over the corresponding beam.

angular resolution of $\sim 1^{\prime \prime}-2^{\prime \prime}$ places only a few independent beams (or "pixels") across the disk, effectively improved resolution of the disk chemistry can be achieved thanks to the fact that the gas kinematics are essentially Keplerian. Beckwith \& Sargent (1993) show that molecular line emission from Keplerian disks displays a "dipole" pattern near the systematic velocity due to the shear created by the orbital motion. The emission near the systemic velocity is dominated by the outer disk regions, and the separation of the emission peaks depends sensitively on the abundance gradient of the emitting species with radius. This important feature, commonly seen in velocity channel maps of disks with a range of inclination angles (even as small as $6^{\circ}-7^{\circ}$ in the case of TW Hydrae; Qi et al. 2004), may be used to study the radial distribution of molecular emission far more precisely than is afforded by the limited number of pixels provided by the available resolution.

Here we report on Submillimeter Array (SMA ${ }^{5}$; Ho et al. 2004) observations of deuterated species in the disk around TW Hydrae, including the first detection and images of $\mathrm{DCN}$ and $\mathrm{DCO}^{+}$. In $\S 2$ we describe the observations, while in $\S 3$ we introduce the analysis method used and the molecular distribution parameters derived. We describe the model fitting results and discuss the implications in $\S 4$, and we present a summary and conclusions in $\S 5$.

\section{OBSERVATIONS}

All of the observations of TW Hydrae (R.A. $=11^{\mathrm{h}} 01^{\mathrm{m}} 51.875^{\mathrm{s}}$, decl. $=-34^{\circ} 42^{\prime} 17.155^{\prime \prime}$; J2000.0) were made between 2005

\footnotetext{
5 The SMA is a joint project of the Smithsonian Astrophysical Observatory and the Academia Sinica Institute of Astronomy and Astrophysics and is funded by the Smithsonian Institution and the Academia Sinica.
}

February and 2006 December using the SMA eight-antenna interferometer located atop Mauna Kea, Hawaii. Tables 1 and 2 summarize the observational parameters for the detected and undetected species, respectively. The SMA receivers operate in a double-sideband (DSB) mode with an intermediate-frequency band of 4-6 GHz, which is sent over fiber optic transmission lines to 24 "overlapping" digital correlator "chunks" covering a $2 \mathrm{GHz}$ spectral window. Two settings were used for observing the $\mathrm{HCN} / \mathrm{DCN}$ and $\mathrm{HCO}^{+} / \mathrm{DCO}^{+}$lines: at $265.7-267.7 \mathrm{GHz}$ (lower sideband) the tuning was centered on the $\mathrm{HCN} J=3-2$ line at $265.8862 \mathrm{GHz}$ in chunk S22, while the $\mathrm{HCO}^{+} J=3-2$ line at $267.5576 \mathrm{GHz}$ was simultaneously observed in chunk S02; at 215.4-217.4 GHz (lower sideband) the tuning was centered on the $\mathrm{DCO}^{+} J=3-2$ line at $216.1126 \mathrm{GHz}$ in chunk $\mathrm{S} 16$, while the DCN $J=3-2$ line at $217.2386 \mathrm{GHz}$ was simultaneously observed in chunk S02 (the HDO $3_{1,2}-2_{2,1}$ line at $225.90 \mathrm{GHz}$ was also covered in the upper sideband in chunk S06). Combinations of two array configurations (compact and extended) were used to obtain projected baselines ranging from 6 to $180 \mathrm{~m}$. Cryogenic SIS receivers on each antenna produced system temperatures (DSB) of $200-1400 \mathrm{~K}$ at $260 \mathrm{GHz}$ and $100-200 \mathrm{~K}$ at $210 \mathrm{GHz}$. Observations of ortho- $\mathrm{H}_{2} \mathrm{D}^{+}$and $\mathrm{H}^{13} \mathrm{CO}^{+}$were simultaneously carried out (in dual receiver observational mode) on 2006 December 28 using only the compact array configuration. Three out of eight antennas were equipped with $400 \mathrm{GHz}$ receivers (DSB system temperature between 400 and $800 \mathrm{~K}$ ) and available for observations of the $372.4213 \mathrm{GHz}$ ortho- $\mathrm{H}_{2} \mathrm{D}^{+} 1_{1,0}-1_{1,1}$ line, and all eight antennas were operating with $345 \mathrm{GHz}$ receivers (DSB system temperature between 150 and $300 \mathrm{~K}$ ) for observations of the $346.9985 \mathrm{GHz} \mathrm{H}{ }^{13} \mathrm{CO}^{+} J=4-3$ and $345.796 \mathrm{GHz}$ $\mathrm{CO} J=3-2$ lines. The correlator was configured with a narrow

TABLE 2

Observational Parameters for SMA TW Hydrae (Undetected Species)

\begin{tabular}{|c|c|c|c|}
\hline Parameter & HDO $3_{1,2}-2_{2,1}$ & $\mathrm{o}-\mathrm{H}_{2} \mathrm{D}^{+} 1_{1,0}-1_{1,1}$ & $\mathrm{p}-\mathrm{D}_{2} \mathrm{H}^{+}-1_{0,1}$ \\
\hline 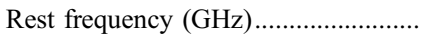 & 225.89672 & 372.42134 & 691.66044 \\
\hline 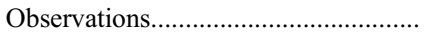 & 2006 Apr 28 & 2006 Dec 28 & 2005 Feb 17 \\
\hline 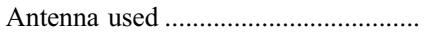 & 8 & 3 & 4 \\
\hline \multirow[t]{2}{*}{ Synthesized beam ................................... } & $5.7^{\prime \prime} \times 3.1^{\prime \prime}$ & $4.7^{\prime \prime} \times 3.8^{\prime \prime}$ & $3.3^{\prime \prime} \times 1.3^{\prime \prime}$ \\
\hline & P.A. $=-0.6^{\circ}$ & P.A. $=14.5^{\circ}$ & P.A. $=7.5^{\circ}$ \\
\hline Channel spacing $\left(\mathrm{km} \mathrm{s}^{-1}\right) \ldots \ldots \ldots \ldots \ldots$ & 0.54 & 0.65 & 0.35 \\
\hline 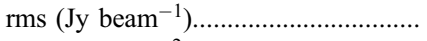 & 0.11 & 1.17 & 7.39 \\
\hline 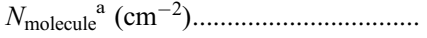 & $<2.0 \times 10^{14}$ & $<1.7 \times 10^{12}$ & $<9.0 \times 10^{14}$ \\
\hline
\end{tabular}

\footnotetext{
${ }^{\text {a }} 1 \sigma$ upper limit.
} 
band of 512 channels over the $104 \mathrm{MHz}$ chunk, which provided $0.2 \mathrm{MHz}$ frequency resolution, or $0.28 \mathrm{~km} \mathrm{~s}^{-1}$ at $217 \mathrm{GHz}$, $0.23 \mathrm{~km} \mathrm{~s}^{-1}$ at $267 \mathrm{GHz}$, and $0.16 \mathrm{~km} \mathrm{~s}^{-1}$ at $372 \mathrm{GHz}$. Observations of the $691.6604 \mathrm{GHz}$ para- $\mathrm{D}_{2} \mathrm{H}^{+} 1_{1,0}-1_{0,1}$ line were shared with observations of the $\mathrm{CO} J=6-5$ line made on 2005 February 17 (details provided in Qi et al. 2006). Calibrations of the visibility phases and amplitudes were achieved with interleaved observations of the quasars J1037-295 and J1147-382, typically at intervals of 20-30 minutes. Observations of Callisto provided the absolute scale for the calibration of flux densities. The uncertainties in the flux scale were estimated to be $15 \%$. All of the calibrations were done using the MIR software package, ${ }^{6}$ while continuum and spectral line images were generated and CLEANed using MIRIAD.

\section{SPECTRAL LINE MODELING}

Several model approaches have been developed to interpret interferometric molecular line and continuum observations from disks (Dutrey et al. 2007). In brief, the approach of Qi et al. (2004, 2006) works as follows: the kinetic temperature and density structure of the disk is determined by modeling the spectral energy distribution assuming well-mixed gas and dust, with the results confirmed by the resolved submillimeter continuum images. Then, a grid of models with a range of disk parameters, including the outer radius $R_{\text {out }}$, the disk inclination $i$, the position angle P.A., and the turbulent line width $v_{\text {turb }}$, are produced and a two-dimensional accelerated Monte Carlo model (Hogerheijde \& van der Tak 2000 ) is used to calculate the radiative transfer and molecular excitation. The collisional rates are taken from the Leiden Atomic and Molecular Database ${ }^{7}$ (Schöier et al. 2005) for non-LTE line radiative transfer calculations. Specifically, in the models used in this paper, the rate coefficients for $\mathrm{HCO}^{+}$and $\mathrm{DCO}^{+}$in collisions with $\mathrm{H}_{2}$ have been calculated by Flower (1999); the rate coefficients for $\mathrm{HCN}$ and DCN in collisions with $\mathrm{H}_{2}$ have been scaled from the rate of $\mathrm{HCN}-\mathrm{He}$ calculated by Green \& Thaddeus (1974). The model parameters are fitted using a $\chi^{2}$ analysis in the $(u, v)$-plane.

In the studies by Qi et al. $(2004,2006)$ the distribution of CO molecules was assumed to follow the $\mathrm{H}$ nuclei as derived from the dust density structure and a gas of solar composition. However, molecules in disks do not necessarily share the same distribution as molecular hydrogen. Theoretical models (e.g., Aikawa et al. 1996; Aikawa \& Nomura 2006) predict so-called threelayered structure; most molecules are photodissociated in the surface layer of the disk and frozen out in the midplane where most hydrogen resides, with an abundance that peaks in the warm molecular layer at intermediate scale heights. To approximate this more complex behavior, we introduce new molecular distribution parameters for use in spectral line modeling.

For first-order analysis in the radial molecular distributions, the column densities are assumed to vary as a power law as a function of radius,

$$
\Sigma_{i}(r)=\Sigma_{i}(10 \mathrm{AU})\left(\frac{r}{10 \mathrm{AU}}\right)^{p_{i}} .
$$

Here $\Sigma_{i}$ and $p_{i}$ describe the column density distribution of a specific species $(i)$, rather than disk (hydrogen) column density. In most disk models to date, a single radial power law is adopted for fitting the hydrogen or dust surface density. By assuming that $\mathrm{CO}$ follows the distribution of the hydrogen column density, Qi

\footnotetext{
${ }^{6}$ Available at http://www.cfa.harvard.edu/ cqi/mircook.html.

7 Available at http://www.strw.leidenuniv.nl/ moldata.
}

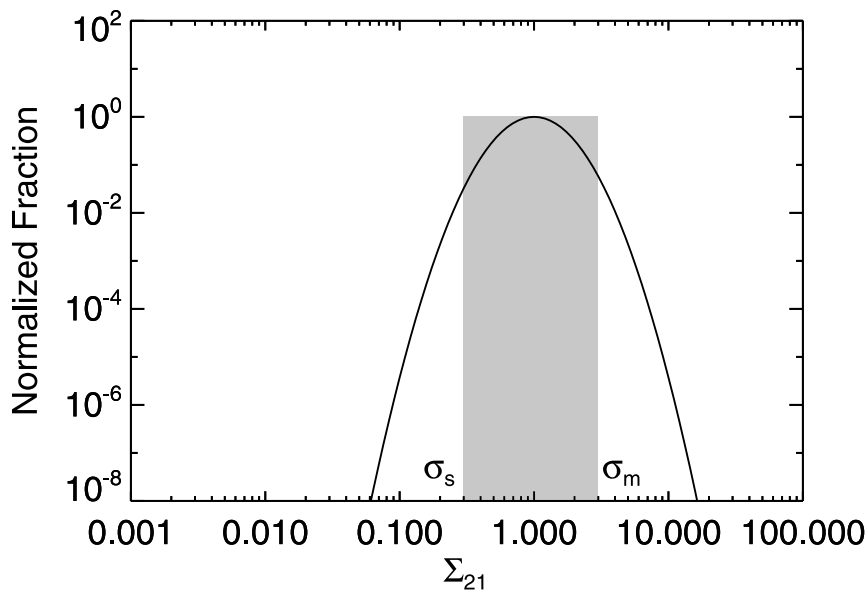

FIG. 1.-Arbitrary molecular vertical distribution as a function of $\Sigma_{21}$ measured from the disk surface at a certain radius. Here $\sigma_{m}$ and $\sigma_{s}$ are the midplane and surface boundary parameters for model fitting ( $\operatorname{see} \S 3$ ).

et al. $(2004,2006)$ also find satisfactory power-law fits for multiple $\mathrm{CO}$ transitions. When a single power law is insufficient for fitting the radial distribution, a broken power law with two different indices will be used.

To calculate the surface density at different radii, the vertical molecular distribution is needed, but this distribution may well vary with distance from the star. However, theoretical models (Aikawa \& Nomura 2006) indicate that the vertical distribution of molecules at different radii is similar as a function of the hydrogen column density measured from the disk surface, $\Sigma_{21} \equiv$ $\Sigma_{\mathrm{H}} /\left(1.59 \times 10^{21} \mathrm{~cm}^{-2}\right)$, where the denominator is the conversion factor of the hydrogen column density to $A_{v}$ for the case of interstellar dust. As indicated by Figure 5 of Aikawa \& Nomura (2006), the vertical distribution of molecular abundances shows a good correlation with $\Sigma_{21}$. Figure 1 shows a schematic diagram of an arbitrary distribution of a molecule in a disk where the $x$-axis shows $\Sigma_{21}$ and the $y$-axis shows the normalized molecular fraction. Smaller $\Sigma_{21}$ values (toward the left of the figure) approach the surface of the disk, while larger $\Sigma_{21}$ values (toward the right) approach the midplane of the disk. For modeling, we make the further simplifying assumption that gaseous molecules exist with a constant abundance in layers between $\sigma_{s}$ and $\sigma_{m}$, the surface and midplane boundaries of $\Sigma_{21}$, respectively, as indicated in Figure 1 (shaded area). While the vertical distribution of molecules in disks may have a more complex distribution than assumed here, the adopted parameters provide a gross approximation to the vertical location where the species is most abundant. This is adequate for a first description, given the quality of the data available. For example, the model of Aikawa \& Nomura (2006) shows two vertical peaks of $\mathrm{HCO}^{+}$, but the secondary peak is an order of magnitude smaller and provides effectively negligible emission. Also, at least for the nearly face-on disk of TW Hydrae, the uncertainties in vertical distributions do not affect the derived radial distribution.

This simple model captures the basic characteristics of threelayered structure predicted by theoretical models. Using the new distribution parameters, $\Sigma_{i}(10 \mathrm{AU})$ and $p_{i}($ radial $)$ and $\sigma_{s}$ and $\sigma_{m}$ (vertical), the radial and vertical distributions of the molecules in disks can be constrained by observation. The best-fit model is obtained by minimizing the $\chi^{2}$, the weighted difference between the real and imaginary part of the complex visibility measured at the selected points of the $(u, v)$-plane. The $\chi^{2}$ values are computed by the simultaneous fitting of channels covering LSR velocities from 

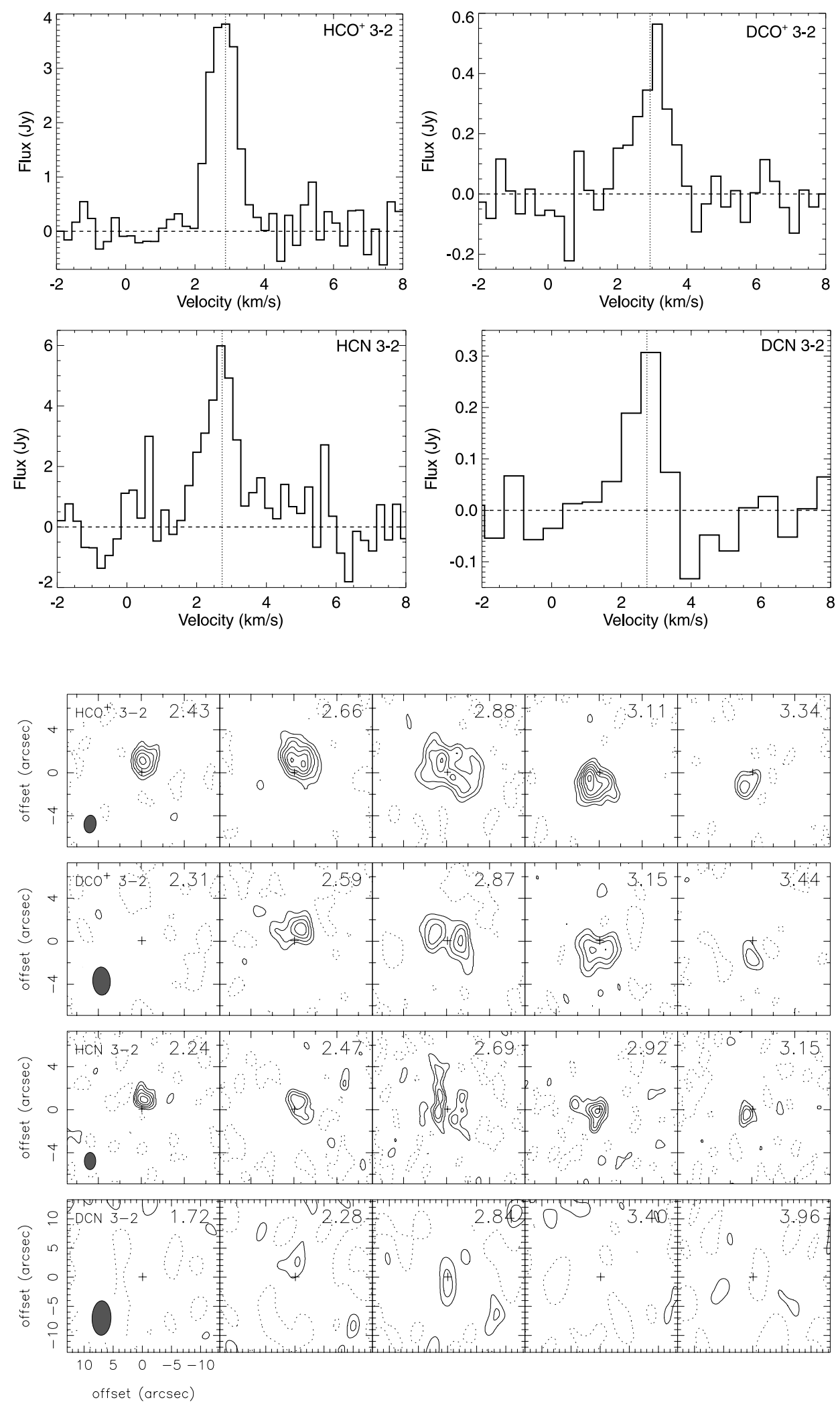

FIG. 2. - Top: $\mathrm{HCO}^{+}, \mathrm{DCO}^{+}, \mathrm{HCN}$, and DCN $J=3-2$ spectra at the peak continuum (stellar) position. The fluxes of $\mathrm{HCO}^{+}$and $\mathrm{DCO}^{+}$are averaged over the beam of $\mathrm{DCO}^{+} J=3-2\left(2.6^{\prime \prime} \times 1.6^{\prime \prime}\right.$, P.A. $\left.=2.8^{\circ}\right)$. The fluxes of $\mathrm{HCN}$ and DCN are averaged over the beam of DCN $J=3-2\left(5.9^{\prime \prime} \times 3.2^{\prime \prime}, \mathrm{P} . \mathrm{A} .=-1.5^{\circ}\right)$. The vertical dotted lines indicate the positions of the fitted $V_{\mathrm{LSR}}$ for each molecular transition, except for DCN 3-2, where we adopt the $V_{\mathrm{LSR}}$ from that of $\mathrm{HCN} J=3-2$. Bottom: Velocity channel maps of the $\mathrm{HCO}^{+}, \mathrm{DCO}^{+}, \mathrm{HCN}$, and $\mathrm{DCN} J=3-2$ emission toward TW Hydrae. The angular resolutions are $1.6^{\prime \prime} \times 1.1^{\prime \prime}$ at P.A. $=-6.3^{\circ}$ for $\mathrm{HCO}^{+} J=3-2$ and $1.6^{\prime \prime} \times 1.1^{\prime \prime}$ at P.A. $=-0.5^{\circ}$ for $\mathrm{HCN} J=3-2$. The cross indicates the continuum (stellar) position. The axes are offsets from the pointing center in arcseconds. The $1 \sigma$ contour steps are $0.4,0.12,0.35$, and $0.09 \mathrm{Jy} \mathrm{beam}^{-1}$ for $\mathrm{HCO}^{+}, \mathrm{DCO}^{+}, \mathrm{HCN}$, and DCN $J=3-2$, respectively, and start at $2 \sigma$. 
TABLE 3

Fitting Results

\begin{tabular}{|c|c|c|c|c|}
\hline Parameter & $\mathrm{HCO}^{+}$ & $\mathrm{DCO}^{+\mathrm{a}}$ & $\mathrm{DCO}^{+\mathrm{b}}$ & $\mathrm{HCN}$ \\
\hline Stellar mass $M_{*}\left(M_{\odot}\right) \ldots \ldots .$. & 0.6 & 0.6 & 0.6 & 0.6 \\
\hline Inclination $i(\mathrm{deg})$ & $6.8 \pm 0.3$ & $7.4 \pm 0.6$ & 7.4 & $6.6 \pm 0.8$ \\
\hline 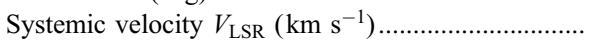 & $2.88 \pm 0.05$ & $2.94 \pm 0.06$ & 2.94 & $2.73 \pm 0.06$ \\
\hline Position angle P.A. (deg) & -27.4 & -27.4 & -27.4 & -27.4 \\
\hline 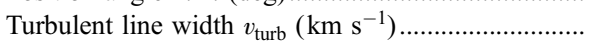 & 0.08 & 0.08 & 0.08 & 0.08 \\
\hline Outer radius $R_{\text {out }}(\mathrm{AU})$ & $200 \pm 10$ & $90 \pm 5$ & 160 & $100 \pm 10$ \\
\hline Column density at $10 \mathrm{AU} \Sigma(10 \mathrm{AU})\left(\mathrm{cm}^{-2}\right) \ldots \ldots \ldots . .$. & $(3.8 \pm 0.5) \times 10^{15}$ & $(1.9 \pm 0.2) \times 10^{10}$ & $4.8 \times 10^{6}$ & $(2.4 \pm 0.4) \times 10^{14}$ \\
\hline Radial power index $p$ & $-2.9 \pm 0.3$ & $2.4 \pm 0.8$ & $7,-6^{\mathrm{c}}$ & $-1.0 \pm 1.2$ \\
\hline 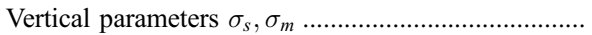 & $0.1,10$ & $0.1,10$ & $0.1,10$ & $0.3,30$ \\
\hline Minimum $\chi^{2} \ldots \ldots \ldots \ldots \ldots \ldots \ldots \ldots$ & 329588 & 575347 & 575347 & 297198 \\
\hline Reduced $\chi^{2} \chi_{r}^{2} \ldots \ldots$ & 1.21 & 1.95 & 1.95 & 1.35 \\
\hline
\end{tabular}

${ }^{\mathrm{a}} \mathrm{DCO}^{+}$model 2.

${ }^{\mathrm{b}} \mathrm{DCO}^{+}$model 3, no error estimation.

${ }^{c} \mathrm{DCO}^{+}$turning point at radius $70 \mathrm{AU}$.

1 to $4 \mathrm{~km} \mathrm{~s}^{-1} ; R_{\mathrm{out}}$ and $p_{i}$ are calculated on grids in steps of 5 (AU) and 0.2 , respectively. We calculate $\log \sigma_{s}$ and $\log \sigma_{m}$ on grids in steps of 0.2 within the range from -2 to 2 , and $\Sigma_{i}(10 \mathrm{AU})$ and $i$ are found with each pair of radial and vertical distribution parameters by minimizing $\chi^{2}$. The systemic velocity $V_{\mathrm{LSR}}$ is fit separately, since it is not correlated with those distribution parameters. For each fit parameter, the $1 \sigma$ uncertainties are estimated as $\chi_{1 \sigma}^{2}=\chi_{m}^{2}+(2 n)^{1 / 2}$, where $n$ is the number of degrees of freedom and $\chi_{m}^{2}$ is the $\chi^{2}$ value of the best-fit model, as in Isella et al. (2007). We tested values for the turbulent velocity in the range from 0.0 to $0.15 \mathrm{~km} \mathrm{~s}^{-1}$ and found that the exact value does not have a significant impact, in part because of the coarse spectral resolution of the data. Therefore, we fixed the turbulent velocity at an intermediate value, $0.08 \mathrm{~km} \mathrm{~s}^{-1}$.

\section{RESULTS AND DISCUSSION}

Figure 2 shows the spectra of $\mathrm{HCO}^{+}, \mathrm{DCO}^{+}, \mathrm{HCN}$, and DCN at the stellar (peak continuum) position and their velocity channel maps. Table 3 summarizes the power-law fitting results on $\mathrm{HCO}^{+}$, $\mathrm{DCO}^{+}$, and $\mathrm{HCN}$. The DCN and $\mathrm{H}^{13} \mathrm{CO}^{+}$lines are too weak for a $\chi^{2}$ analysis of their distribution parameters, and so only the ratios of $\mathrm{DCN} / \mathrm{HCN}$ and $\mathrm{HCO}^{+} / \mathrm{H}^{13} \mathrm{CO}^{+}$are fit. Figure 3 shows the

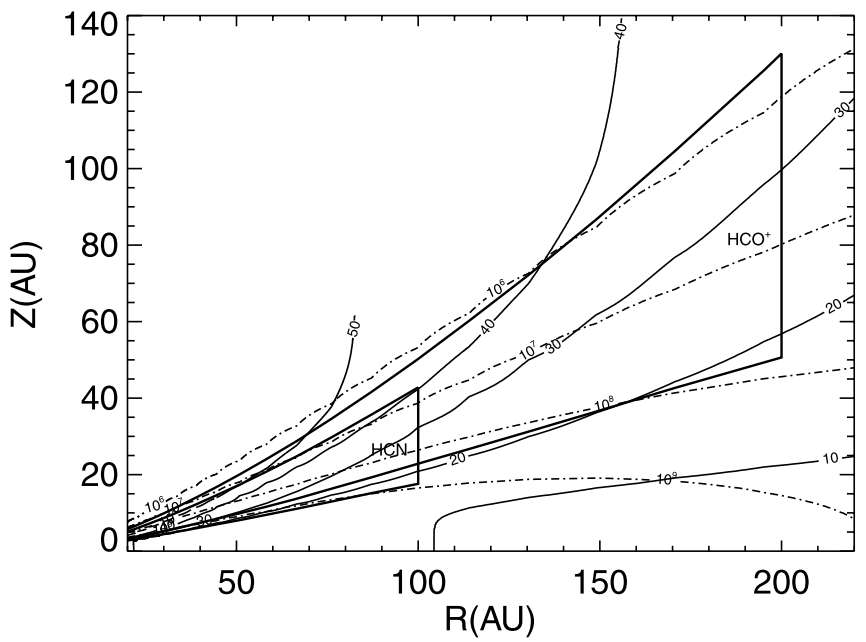

FIG. 3.-Solid and dot-dashed contours show the temperature and density profiles from the TW Hydrae model of Calvet et al. (2002). The large and small triangular regions enclose the locations of $\mathrm{HCO}^{+}$and $\mathrm{HCN}$ in the model (see text). [See the electronic edition of the Journal for a color version of this figure.] density and temperature contours of the disk model (adopted from Calvet et al. 2002) and the locations of $\mathrm{HCO}^{+}$and $\mathrm{HCN}$ derived from the model fitting procedure. Figure 4 shows the radial distribution of the molecular column densities of the best-fit models for $\mathrm{HCO}^{+}, \mathrm{DCO}^{+}$, and $\mathrm{HCN}$. Not surprisingly, we found that the radial distributions are better constrained than the vertical ones. The local minima of $\chi^{2}$ for different grids of vertical parameters are within the noise limit; i.e., the vertical parameters are the least well determined, due in part to the face-on nature of the TW Hydrae disk. We therefore treat the best-fit vertical results as fixed and investigate the uncertainty of other parameters.

\section{1. $\mathrm{HCO}^{+}$and $\mathrm{DCO}^{+}$}

The first detection of $\mathrm{DCO}^{+}$in the disk around TW Hydrae was obtained by van Dishoeck et al. (2003) with the James Clerk Maxwell Telescope (JCMT), who reported a beam-averaged $\mathrm{DCO}^{+} / \mathrm{HCO}^{+}$abundance ratio of 0.035 . Our spatially resolved observations of the $\mathrm{DCO}^{+}$and $\mathrm{HCO}^{+} J=3-2$ emission from the disk suggest a more complex chemical situation.

Figure 5 shows the channel maps of $\mathrm{HCO}^{+} J=3-2$, together with the best-fit model and the data-model residuals. Table 3 lists the best-fit model parameters. The $\chi^{2}$ surface for the radial power index $p_{\mathrm{HCO}^{+}}$and the outer radius $R_{\text {out }}$ is shown in Figure 6 (top). The $1 \sigma$ contour confines $R_{\text {out }}$ to $200 \pm 10 \mathrm{AU}$ and $p_{\mathrm{HCO}^{+}}$to

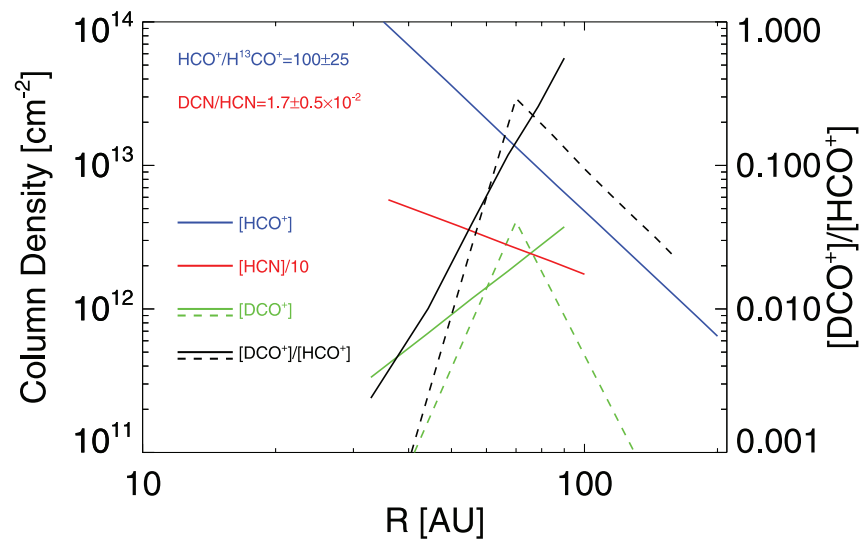

FIG. 4.- Radial distribution of molecular column densities and $\mathrm{DCO}^{+} / \mathrm{HCO}^{+}$ ratio for the best-fit models for TW Hydrae. The solid lines depict single power-law fits, while the dashed lines depict $\mathrm{DCO}^{+}$model 3, where two power-law indices are used. 


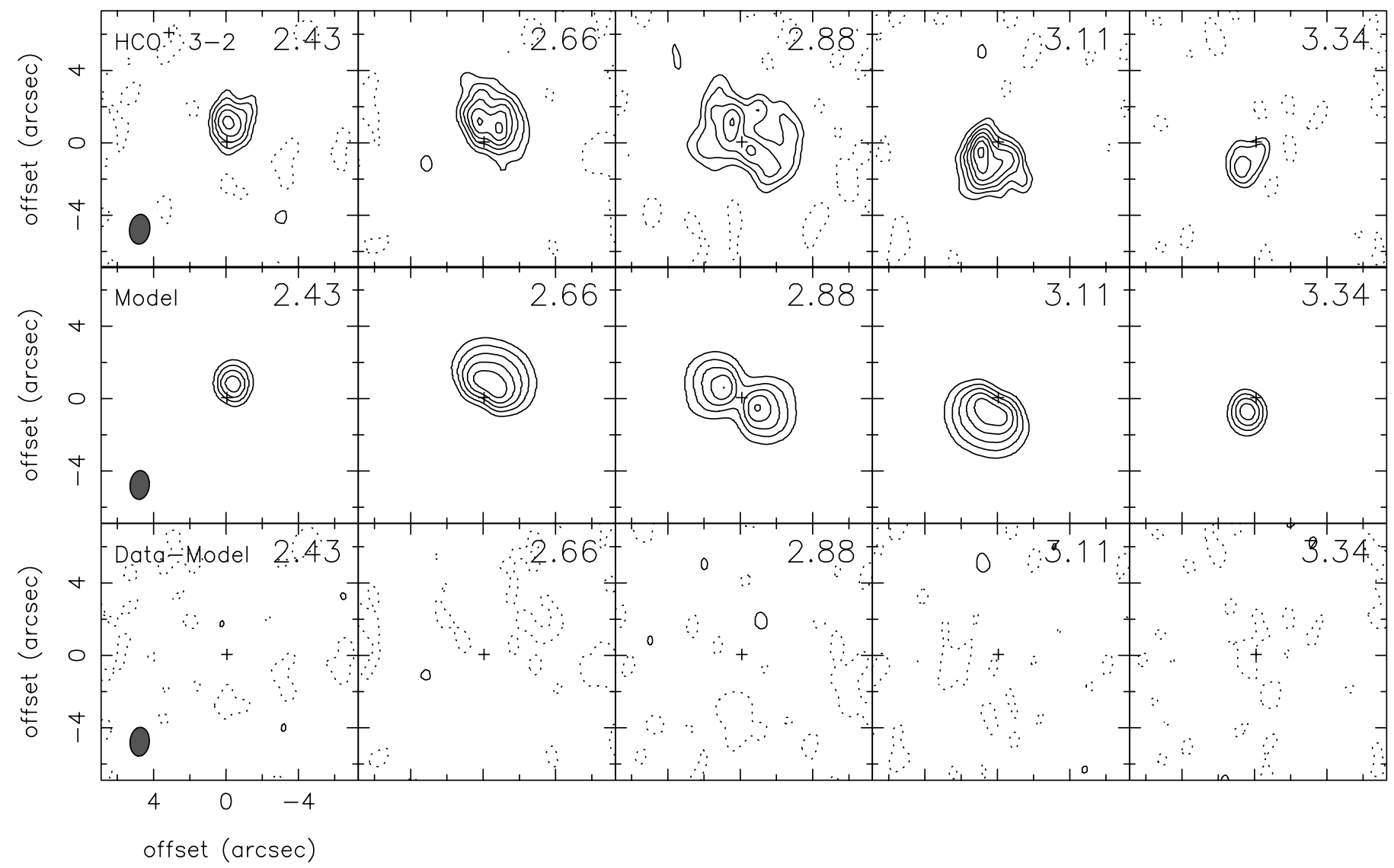

Fig. 5. - Top panels: Velocity channel maps of the $\mathrm{HCO}^{+} J=3-2$ emission toward TW Hydrae. The angular resolution is $1.6^{\prime \prime} \times 1.1^{\prime \prime}$ at P.A. $=-6.3^{\circ}$. The cross indicates the continuum (stellar) position. The axes are offsets from the pointing center in arcseconds. The $1 \sigma$ contour step is $0.4 \mathrm{Jy}_{\text {beam }}{ }^{-1}$, and the contours start at $2 \sigma$. Middle panels: Channel map of the best-fit model with the same contour levels. Bottom panels: Difference between the best-fit model and data on the same contour scale.

$-2.9 \pm 0.3$. The $\mathrm{H}^{13} \mathrm{CO}^{+} J=4-3$ line was also detected, but the emission was not strong enough to constrain its distribution. Assuming that $\mathrm{H}^{13} \mathrm{CO}^{+}$has the same distribution as $\mathrm{HCO}^{+}$, then fitting the ratio of $\mathrm{HCO}^{+} / \mathrm{H}^{13} \mathrm{CO}^{+}$to match the intensity of the $\mathrm{H}^{13} \mathrm{CO}^{+}$emission, indicates an $\mathrm{HCO}^{+} / \mathrm{H}^{13} \mathrm{CO}^{+}$ratio of $100 \pm 25$. This value is consistent with the nominal solar system value of 89 . Figure 7 presents the best-fit model spectra of $\mathrm{H}^{13} \mathrm{CO}^{+} J=4-3$ overlaid on the SMA data.

The vertical distribution of $\mathrm{DCO}^{+}$is even less well constrained than that of $\mathrm{HCO}^{+}$, and we choose to adopt the same values of $\sigma_{s}$ and $\sigma_{m}$ for both $\mathrm{HCO}^{+}$and $\mathrm{DCO}^{+}$(as indicated in Table 3). Because of the nearly face-on viewing geometry for the disk, the fitting of radial distribution power-law index $p_{i}$ is not affected significantly by the uncertainties in vertical structure, but only the value of $\Sigma_{i}(10 \mathrm{AU})$ needs to be adjusted.

Examination of the channel maps shows on inspection that the radial distributions of $\mathrm{DCO}^{+}$and $\mathrm{HCO}^{+}$are different. To demonstrate how differences in the radial distribution affect the resulting images, Figure 8 presents three models of $\mathrm{DCO}^{+}$radial distribution and Figure 9 shows the corresponding simulated channel maps of the $\mathrm{DCO}^{+} J=3-2$ line.

Model 1 assumes that the $\mathrm{DCO}^{+}$distribution follows the best-fit model of $\mathrm{HCO}^{+}$. The minimum $\chi^{2}$ is determined to be 575,396 , and the corresponding $\mathrm{DCO}^{+} / \mathrm{HCO}^{+}$is found to be $4.7 \times 10^{-2}$. Comparing the simulated maps from model 1 with the data in Figure 9 shows a distinct difference in that the double-peaked nature of the central channel in the data is more obvious than in this model. The contrast of the contour levels between the central channel and the adjacent channels is also smaller in the data than in this model. Because the emission of the central channel mostly originates at large disk radii, these differences suggest that the $\mathrm{DCO}^{+}$emission arising from the outer regions of the disk is stronger than predicted by this model. The slope of the radial $\mathrm{DCO}^{+}$distribution does not decrease as steeply as that of $\mathrm{HCO}^{+}$. In other words, the $\mathrm{D} / \mathrm{H}$ ratio must increase with increasing radius.

Model 2 shows the best-fit result for the radial distribution of $\mathrm{DCO}^{+}$assuming a single power index. As shown in Figure 4, the radial distribution of $\mathrm{DCO}^{+}$is strikingly different from that of $\mathrm{HCO}^{+}$, with a positive power index of 2.4 and a smaller but better constrained $R_{\text {out }}=90 \pm 5 \mathrm{AU}$. The contours of the iso- $\chi^{2}$ surface for $\mathrm{DCO}^{+}$in Figure 6 (middle panel) indicate that the uncertainty of the radial power index is very large but that the index is still larger than 1.6 within the $1 \sigma$ error, much larger than -2.9 found for $\mathrm{HCO}^{+}$. The simulated $\mathrm{DCO}^{+}$channel maps for model 2 shown in Figure 9 are an improvement over model 1 in matching the data. However, in this model, $R_{\text {out }}(\sim 90 \mathrm{AU})$ is much smaller than the disk radius observed with $\mathrm{HCO}^{+}$and $\mathrm{CO}$ ( $\sim 200 \mathrm{AU})$, and $\mathrm{DCO}^{+}$increases with radius but then disappears sharply at $R_{\text {out }} \sim 90 \mathrm{AU}$, as a step function, which is hard to understand. Comparison with the data shows that there are fewer complete contours around the double peaks in the central channel, which suggests that the maximum $\mathrm{DCO}^{+}$emission is at an intermediate radius rather than at the edge.

For model 3 , instead of using a single power index $(p)$ to fit for the radial distribution of $\mathrm{DCO}^{+}$column density $N\left(\mathrm{DCO}^{+}\right)$, the fit uses two power indices ( $p 1$ and $p 2)$ and a turning point $\left(R_{t}\right)$ where the power-law index changes from $p 1$ to $p 2$, i.e., the location of the peak of $N\left(\mathrm{DCO}^{+}\right)$. The parameters $p 1, p 2$, and $R_{t}$ are searched 

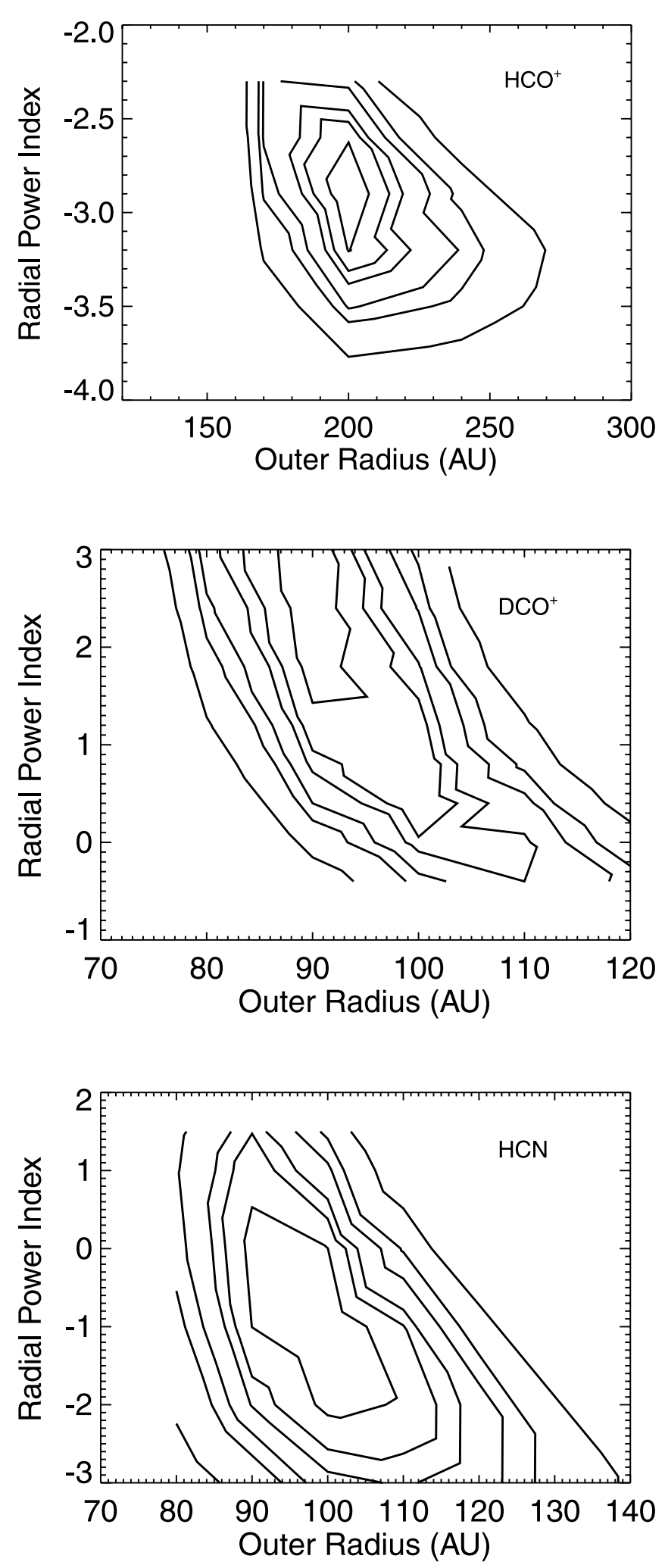

FIG. 6.-Iso- $\chi^{2}$ surfaces of $R_{\text {out }}, p_{i}$ for $\mathrm{HCO}^{+}, \mathrm{DCO}^{+}$(as in model 2), and $\mathrm{HCN}$. Contours correspond to the $1-6 \sigma$ errors. $\mathrm{For}_{\mathrm{DCO}^{+}}$, the index values larger than 3 at around $90 \mathrm{AU}$ indicate the ratios of $\mathrm{DCO}^{+} / \mathrm{HCO}^{+}$larger than 1 , so the $\chi^{2}$ surface is not calculated beyond that.

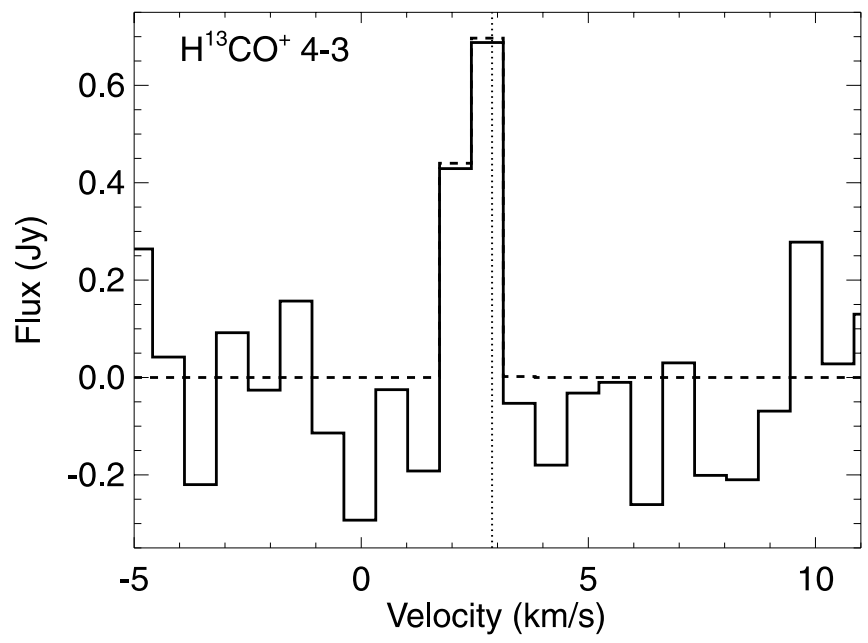

FIG. 7.-Beam-averaged $\mathrm{H}^{13} \mathrm{CO}^{+} J=4-3$ spectra at the continuum (stellar) position. The SMA data are indicated by the solid histogram and the simulated model by the dashed histogram. The vertical dotted line indicates the position of the fitted $V_{\mathrm{LSR}}$ for $\mathrm{HCO}^{+} J=3-2$. [See the electronic edition of the Journal for a color version of this figure.]

within limited grids to minimize $\chi^{2}$. In this model, $N\left(\mathrm{DCO}^{+}\right)$is found to increase with radius out to $\sim 70 \mathrm{AU}$ and then decrease. Table 3 presents the best-fit parameters; no error estimation is provided due to the computation difficulties. Figure 9 shows the best-fit model images. Comparison of model 3 with the data shows a visual improvement over model 2 in the central channel, although the $\chi^{2}$ values of the two models are not distinguishable. We thus cannot clearly discriminate with the $\chi^{2}$ statistic whether there is indeed a peak with radius for $N\left(\mathrm{DCO}^{+}\right)$as in model 3 or $\mathrm{DCO}^{+}$increases with radius and disappears suddenly around $90 \mathrm{AU}$ as in model 2. Even with this ambiguity, however, both models imply that the $\mathrm{D} / \mathrm{H}$ ratios change by at least an order of magnitude (0.01-0.1) from radii $<30$ to $\geq 70 \mathrm{AU}$ and that there is a rapid falloff of $N\left(\mathrm{DCO}^{+}\right)$at radii larger than $90 \mathrm{AU}$. Because the emission in the central channel comes from the outer part of the disk (Keplerian rotation) projected along the line of sight with a very small inclination of around $7^{\circ}$ for TW Hydrae, the central velocity channel is most important for constraining the radial distribution. Based on the difference in the central velocity channel map between the models, we believe model 3 is the more plausible description of the radial distribution of $\mathrm{DCO}^{+}$. Figure 10

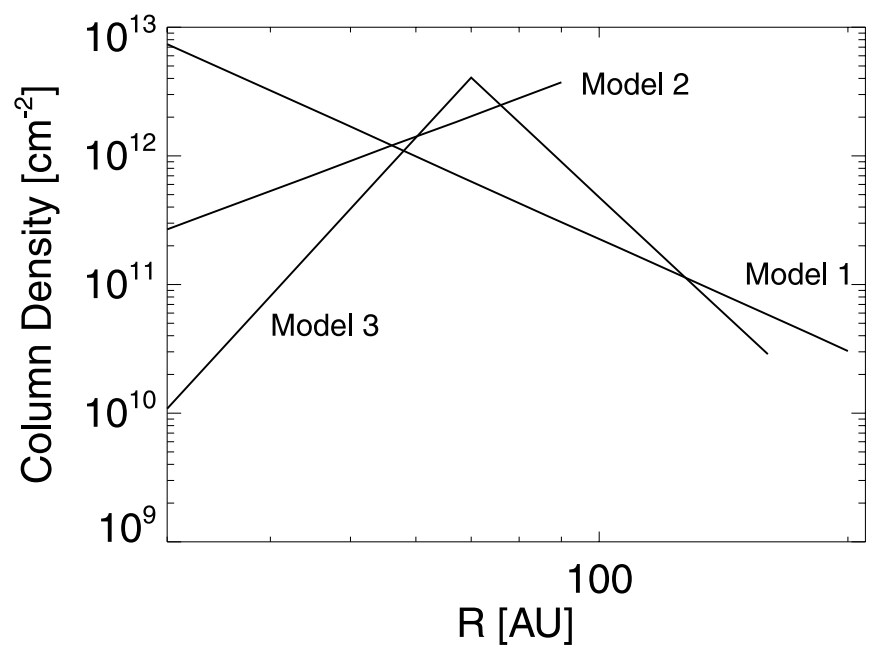

FIG. 8.-Models of $\mathrm{DCO}^{+}$with different distributions of radial column densities. [See the electronic edition of the Journal for a color version of this figure.] 


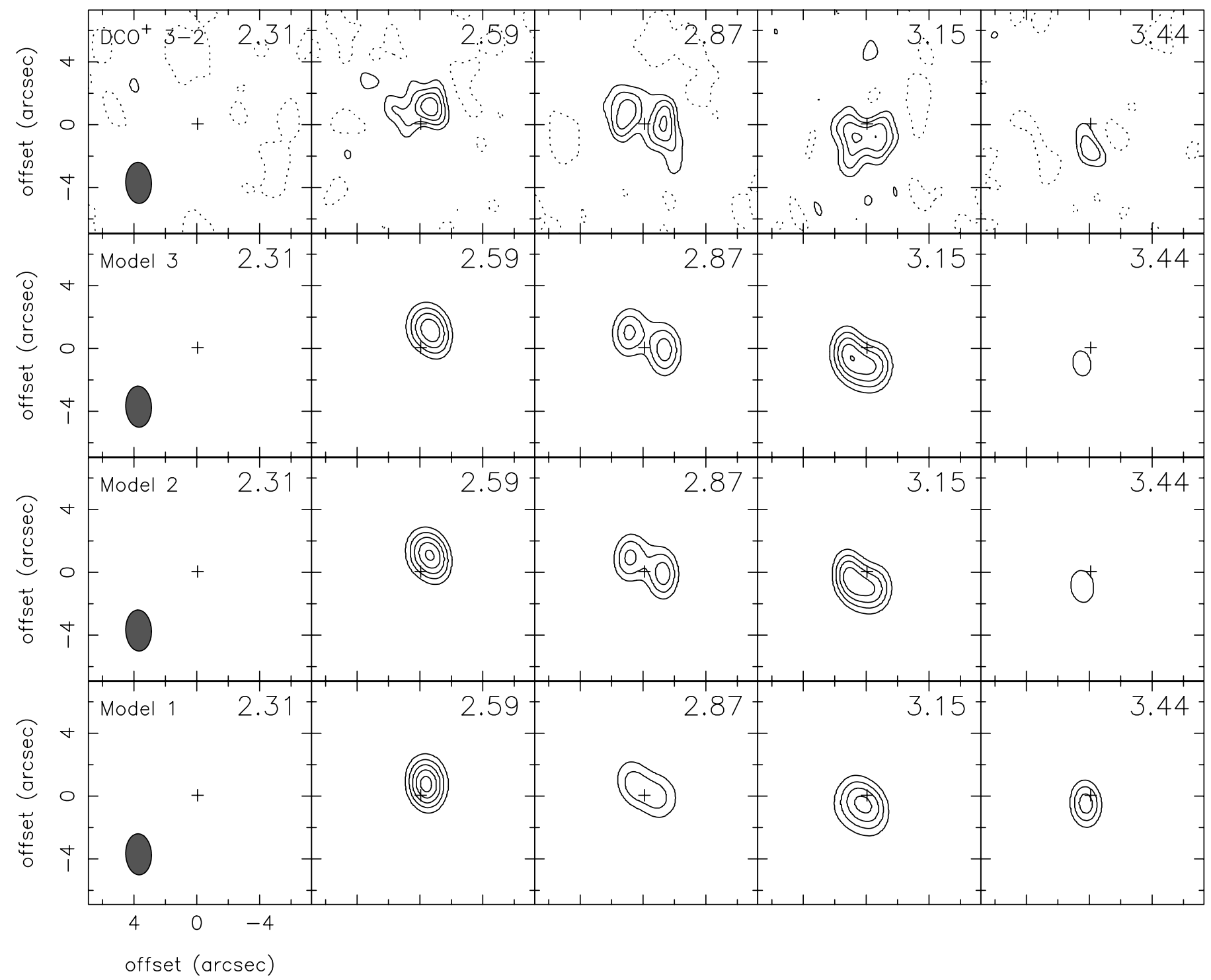

FIG. 9.- $-\mathrm{DCO}^{+} J=3-2$ channel maps toward TW Hydrae and the simulated model distributions depicted in Fig. 8 .

shows the channel maps of the $\mathrm{DCO}^{+} J=3-2$ emission, together with those of model 3 and the data-model residuals.

Observations of deuterated molecular ions and the level of deuterium fractionation have been used to estimate the ionization degree in molecular clouds, and a similar analysis can be applied to circumstellar disks. If we consider only the ionization balance determined by $\mathrm{HCO}^{+}, \mathrm{H}_{3}^{+}, \mathrm{DCO}^{+}$, and electrons in steady state, as shown in equation (14) of Caselli (2002), the electron fractional abundance can be derived to be around $10^{-7}$. Of course, this value is only valid in the intermediate layer where $\mathrm{HCO}^{+}$is abundant and multiply deuterated $\mathrm{H}_{3}^{+}$is less abundant than $\mathrm{HCO}^{+}$. Several important complications are also neglected in this analysis, including the presence of other atomic and molecular ions, neutral species besides $\mathrm{CO}$ which destroy $\mathrm{H}_{2} \mathrm{D}^{+}$, and negatively charged dust grains and refractory metals. Still, accurate measurements of $\mathrm{DCO}^{+}$and $\mathrm{HCO}^{+}$are the first steps toward an understanding of the ionization fraction in the disk.

The increase of $\mathrm{D} / \mathrm{H}$ ratios from the inner to outer disk is generally consistent with current theoretical models of the gasdeuterium fractionation processes that consider the effect of cold temperatures. But the quick disappearance of $\mathrm{DCO}^{+}$at radii beyond $90 \mathrm{AU}$ (compared with $R_{\text {out }}$ around $200 \mathrm{AU}$ for $\mathrm{CO}$ and
$\mathrm{HCO}^{+}$) is puzzling, since $\mathrm{DCO}^{+}$is expected to be abundant and observable in the cold outer region of the disk where $\mathrm{HCO}^{+}$is still available. More theoretical work is needed to explain the disappearance of $\mathrm{DCO}^{+}$in the outer part of the disk.

\section{2. $\mathrm{HCN}$ and $\mathrm{DCN}$}

Figure 11 shows the $\mathrm{HCN} J=3-2$ channel maps, together with the best-fit model and residuals. Table 3 lists the best-fit model parameters, and Figure 4 shows the radial distribution of the column density derived. The $\chi^{2}$ surface shown in Figure 6 (bottom panel) indicates $R_{\mathrm{out}}=100 \pm 10 \mathrm{AU}$ and $p_{\mathrm{HCN}}=$ $-1.0 \pm 1.2$. The radial power index is poorly constrained, probably due to more complex distributions for HCN. A detailed comparison of the molecular distributions of $\mathrm{HCN}$ and $\mathrm{CN}$ will be presented elsewhere.

Although the best-fit vertical parameters seem to indicate that $\mathrm{HCN}\left(\sigma_{m, \mathrm{HCN}}=30\right)$ is found much deeper toward the midplane than is $\mathrm{HCO}^{+}\left(\sigma_{m, \mathrm{HCO}^{+}}=10\right)$, we emphasize that we are not able to accurately constrain the vertical distributions from the present data. This ambiguity strongly affects the column density of $\mathrm{HCN}$ [i.e., $\left.\Sigma_{\mathrm{HCN}}(10 \mathrm{AU})\right]$ needed to fit the data (not the power index $p_{\mathrm{HCN}}$ of radial distribution). A worse fit to the data $\left(\chi^{2}\right.$ larger by 


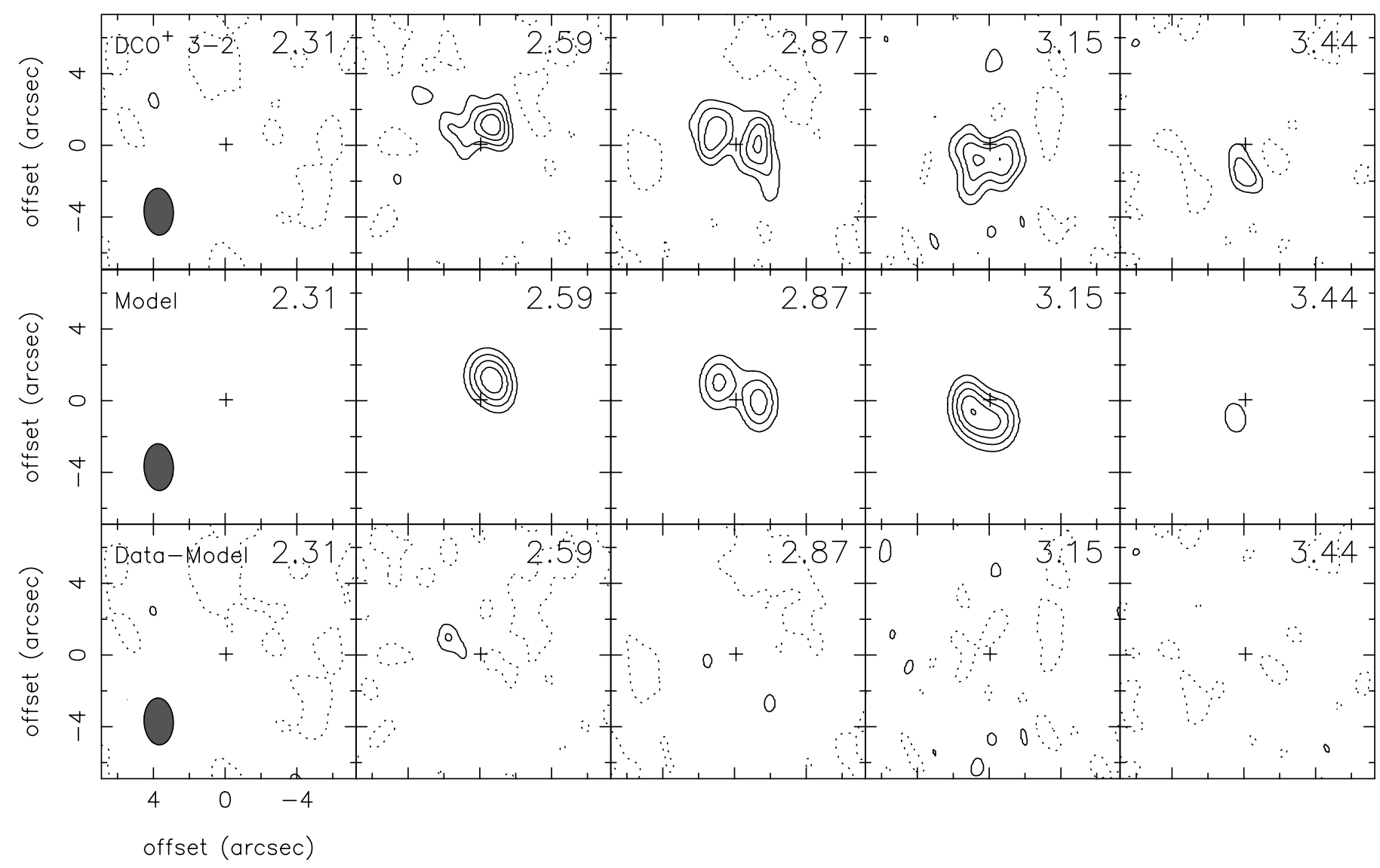

Fig. 10.-Top panels: Velocity channel map of the $\mathrm{DCO}^{+} J=3-2$ emission toward TW Hydrae. The angular resolution is $2.6^{\prime \prime} \times 1.6^{\prime \prime}$ at P.A. $=2.8^{\circ}$. The cross indicates the continuum (stellar) position. The axes are offsets from the pointing center in arcseconds. The $1 \sigma$ contour step is $0.12 \mathrm{Jy}^{\text {beam }}{ }^{-1}$, and the contours start at $2 \sigma$. Middle panels: Channel map of model 3 with the same contour levels. Bottom panels: Difference between model 3 and data on the same contour scale.

$3 \sigma$ over the best-fit model) can be obtained by assuming that the vertical distributions of $\mathrm{HCN}$ and $\mathrm{HCO}^{+}$are the same, but the $\mathrm{HCN}$ column density is 1.5 times larger than that needed for the best-fit model due to higher density near the midplane.

The DCN $J=3-2$ transition is detected at a signal-to-noise ratio of 3 near the fitted HCN $V_{\text {LSR }}$ of $2.73 \mathrm{~km} \mathrm{~s}^{-1}$ (Fig. 2). While this signal-to-noise ratio is not high, the significance of the detection is further supported by the channel maps (Fig. 12, top), where the velocity gradient along the disk position angle of approximately $-30^{\circ}$ is consistent with that seen in $\mathrm{CO} J=2-1$ and $J=3-2$ (Qi et al. 2004) and the other molecular lines presented in this paper. Since the DCN 3-2 emission is weak, we are not able to fit for the molecular distribution and so make the simplifying assumption that the distribution of $\mathrm{DCN}$ follows that of $\mathrm{HCN}$. As with $\mathrm{H}^{13} \mathrm{CO}^{+}$, we fit the DCN/HCN ratio over the whole disk and determine it to be $(1.7 \pm 0.5) \times 10^{-2}$. To again emphasize the impact of the assumed vertical distribution on the derived column densities, the $\mathrm{DCN} / \mathrm{HCN}$ ratio could be as high as $5 \times 10^{-2}$ if HCN and DCN are distributed vertically over the same region as $\mathrm{HCO}^{+}$.

Highly fractionated DCN/HCN ratios have been measured in comets. In the coma of comet Hale-Bopp, for example, Meier et al. (1998) reported the ratio to be around $2.3 \times 10^{-3}$, but higher $\mathrm{DCN} / \mathrm{HCN}$ ratios, $(\mathrm{D} / \mathrm{H})_{\mathrm{HCN} \text {,jet }} \approx 0.025$, are detected from the pristine material sublimed from icy grains ejected in jets from the nucleus which may present a more representative sampling of cometary ices that have not experienced significant thermal processing (Blake et al. 1999). Such ratios are consistent with those found here in the TW Hydrae disk, indicating that high $\mathrm{D} / \mathrm{H}$ ratios in comets could originate from material in the outer regions of disks where in situ deuterium fractionation is ongoing, rather than requiring an inheritance from interstellar material.

\subsection{Upper Limits for $\mathrm{H}_{2} \mathrm{D}^{+}, \mathrm{D}_{2} \mathrm{H}^{+}$, and $\mathrm{HDO}$}

In the disk midplane, $\mathrm{H}_{2}$ is expected to be gaseous, and the molecular ion formed by the cosmic-ray ionization of $\mathrm{H}_{2}, \mathrm{H}_{3}^{+}$, is expected to be the most abundant ion. Unfortunately, $\mathrm{H}_{3}^{+}$is only detectable in cold gas via infrared absorption. In its deuterated forms, however, $\mathrm{H}_{2} \mathrm{D}^{+}$and even $\mathrm{D}_{2} \mathrm{H}^{+}$are expected to be abundant in the cold, dense gas (Ceccarelli \& Dominik 2005). The ground-state transition of ortho- $\mathrm{H}_{2} \mathrm{D}^{+}$was first detected in a young stellar object (NGC 1333, IRAS 4A) by Stark et al. (1999) and in a prestellar core (L1544) by Caselli et al. (2003). Both $\mathrm{H}_{2} \mathrm{D}^{+}$and $\mathrm{D}_{2} \mathrm{H}^{+}$have been detected toward another prestellar core, $16293 \mathrm{E}$, via their ground-state submillimeter rotational lines (Vastel et al. 2004). The inclusion of multiply deuterated $\mathrm{H}_{3}^{+}$in chemical models leads to predictions of higher values of the $\mathrm{D} / \mathrm{H}$ ratio in cold, high-density regions of the interstellar medium. Similarly, in the dense, cold disk midplane, $\mathrm{CO}$ is depleted, and high abundances of $\mathrm{H}_{2} \mathrm{D}^{+}$and $\mathrm{D}_{2} \mathrm{H}^{+}$are expected. For this reason, Ceccarelli et al. (2004) searched for the ground-state transition of ortho- $\mathrm{H}_{2} \mathrm{D}^{+}$ with the Caltech Submillimeter Observatory and reported 3.2 and $4.7 \sigma$ detections toward TW Hydrae and DM Tauri, respectively. With the 400 and $690 \mathrm{GHz}$ receiver-equipped SMA antennas, we searched for the $372 \mathrm{GHz}$ ortho- $\mathrm{H}_{2} \mathrm{D}^{+} 1_{1,1}-1_{1,0}$ and $692 \mathrm{GHz}$ para- $\mathrm{D}_{2} \mathrm{H}^{+} 1_{1,0}-1_{0,1}$ lines toward TW Hydrae. No significant emission signals were detected. Here we discuss the upper limits and their implications. 


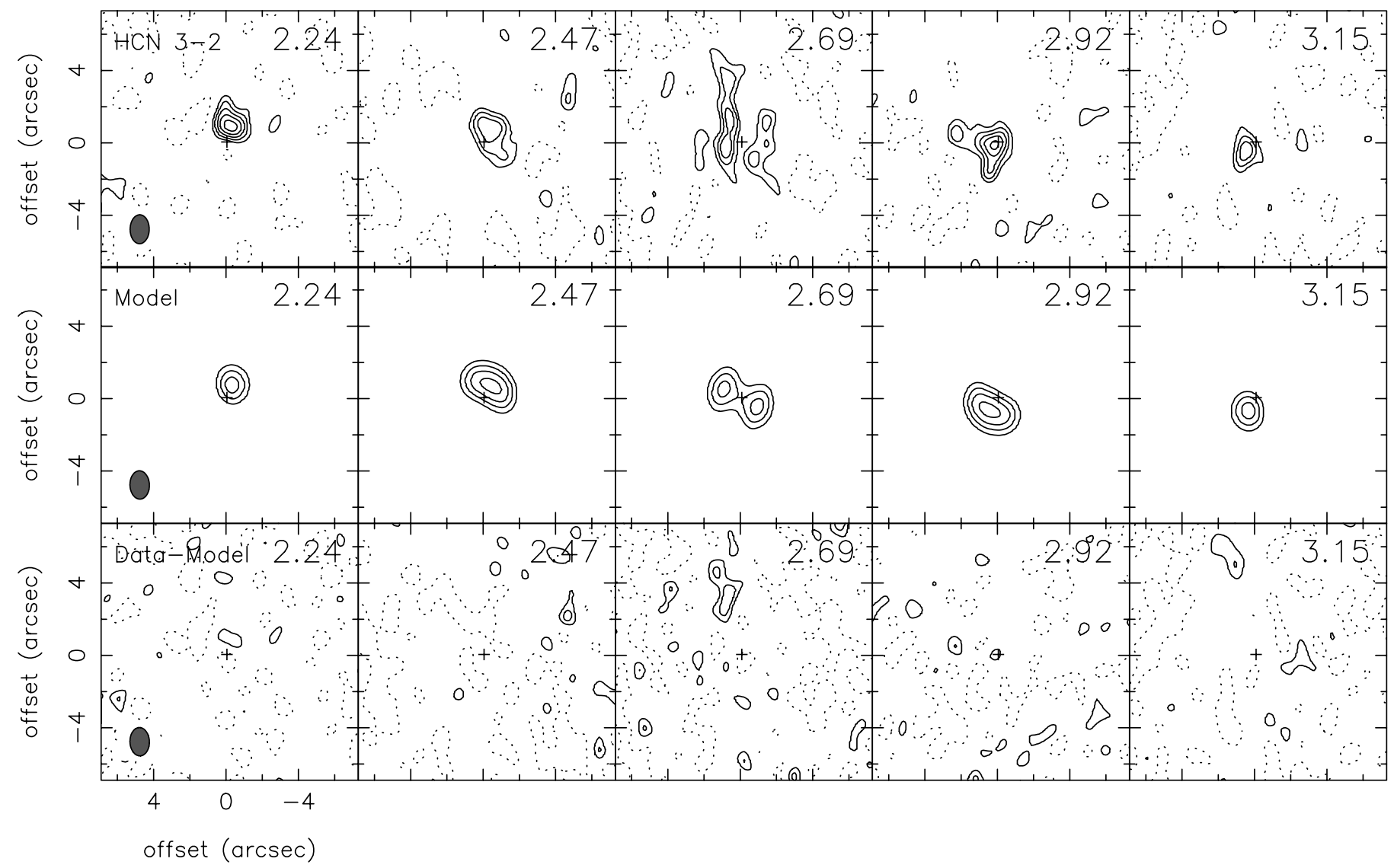

Fig. 11.-Top panels: Velocity channel map of the HCN $J=3-2$ emission toward TW Hydrae. The angular resolution is $1.6^{\prime \prime} \times 1.1^{\prime \prime}$ at P.A. $=-0.5^{\circ}$. The cross indicates the continuum (stellar) position. The axes are offsets from the pointing center in arcseconds. The $1 \sigma$ contour step is 0.35 Jy beam $^{-1}$, and the contours start at $2 \sigma$. Middle panels: Channel map of the best-fit model with the same contour levels. Bottom panels: Difference between the best-fit model and data on the same contour scale.

Our three-antenna SMA observations give a $1 \sigma$ upper limit for the ortho- $\mathrm{H}_{2} \mathrm{D}^{+} 1_{1,1}-1_{1,0}$ line emission of $1.2 \mathrm{Jy}_{\text {beam }}^{-1} \mathrm{~km} \mathrm{~s}^{-1}$ with a $4.7^{\prime \prime} \times 3.8^{\prime \prime}$ synthesized beam. To compare the result with single-dish data, the extent of the source emission must be known. Since $\mathrm{H}_{2} \mathrm{D}^{+}$was observed along with the $\mathrm{H}^{13} \mathrm{CO}^{+} 4-3$ line in a dual receiver observation on 2006 December 28, and $\mathrm{H}^{13} \mathrm{CO}^{+} 4-3$ has been clearly detected at JCMT (van Dishoeck et al. 2003), we can compare the intensities of these two lines between the SMA data and the single-dish observations to constrain the emitting areas. The $\mathrm{H}^{13} \mathrm{CO}^{+} 4-3$ line was detected at JCMT with an integrated intensity of $0.07 \mathrm{~K} \mathrm{~km} \mathrm{~s}^{-1}$ in a $13^{\prime \prime}$ beam (van Dishoeck et al. 2003), while for the SMA the integrated intensity of this line is determined to be $0.61 \mathrm{Jy} \mathrm{beam}^{-1} \mathrm{~km} \mathrm{~s}^{-1}$ with a beam of $4.1^{\prime \prime} \times 1.8^{\prime \prime}$. If the extent of the emission of $\mathrm{H}^{13} \mathrm{CO}^{+}$is similar to that of $\mathrm{H}_{2} \mathrm{D}^{+}$, our interferometric upper limit of $\mathrm{Jy} \mathrm{beam}^{-1} \mathrm{~km} \mathrm{~s}^{-1}$ for $\mathrm{H}_{2} \mathrm{D}^{+}$(considering the small change of the beam sizes) becomes $0.09 \mathrm{~K} \mathrm{~km} \mathrm{~s}^{-1}(1 \sigma)$ or $0.27 \mathrm{~K} \mathrm{~km} \mathrm{~s}^{-1}$ $(3 \sigma)$, which is consistent with the $2 \sigma$ upper limit of about $0.2 \mathrm{~K} \mathrm{~km} \mathrm{~s}^{-1}$ by the JCMT (Thi et al. 2004) but slightly lower than the $3.2 \sigma$ detection of $0.39 \mathrm{~K} \mathrm{~km} \mathrm{~s}^{-1}$ by Ceccarelli et al. (2004). We estimate the $1 \sigma$ upper limit of the ortho- $\mathrm{H}_{2} \mathrm{D}^{+}$column density to be $1.7 \times 10^{12} \mathrm{~cm}^{-2}$, according to equation (4) of Vastel et al. (2004), which is slightly less than the $2 \sigma$ upper limit estimate of $4.4 \times 10^{12} \mathrm{~cm}^{-2}$ by Thi et al. (2004), since the SMA data had a smaller noise level. Of course, this analysis assumes that the extent of $\mathrm{H}_{2} \mathrm{D}^{+}$is similar to that of $\mathrm{H}^{13} \mathrm{CO}^{+}$. With the deployment of more $400 \mathrm{GHz}$ receivers on the SMA and further $\mathrm{H}_{2} \mathrm{D}^{+}$observations, it should be possible to provide better constraints on the $\mathrm{H}_{2} \mathrm{D}^{+}$abundance in the disk.
We estimate the $1 \sigma$ upper limit for para- $\mathrm{D}_{2} \mathrm{H}^{+} 1_{1,0}-1_{0,1}$ to be $5.35 \mathrm{Jy} \mathrm{beam}^{-1} \mathrm{~km} \mathrm{~s}^{-1}$ with a beam of $3.3^{\prime \prime} \times 1.3^{\prime \prime}$. The $1 \sigma$ upper limit to the para- $\mathrm{D}_{2} \mathrm{H}^{+}$column density is estimated to be $9.0 \times 10^{14} \mathrm{~cm}^{-2}$. This is less constrained than $\mathrm{H}_{2} \mathrm{D}^{+}$due to the relatively poor system sensitivity at $690 \mathrm{GHz}$.

For HDO $3_{1,2}-2_{2,1}$, the $1 \sigma$ upper limit is $0.10 \mathrm{Jy} \mathrm{beam}^{-1} \mathrm{~km} \mathrm{~s}^{-1}$. Assuming an excitation temperature of $30 \mathrm{~K}$, the upper limit for the HDO column density is $2.0 \times 10^{14} \mathrm{~cm}^{-2}$. Since the lower state energy level of this line is nearly $160 \mathrm{~K}$, it must originate from warm regions of the disk, which are quite distinct from the cold layers where HDO ground-state transition absorption, as found in DM Tauri (Ceccarelli et al. 2005), must arise, although we note that the detection of the HDO absorption line in DM Tauri has been disputed by Guilloteau et al. (2006).

\section{SUMMARY AND CONCLUSIONS}

Observations of deuterated species in circumstellar disks are important for understanding the origin of primitive solar system bodies in that they can directly constrain the deuterium fractionation in the outer regions where cometary ices are likely formed. Spatially resolved observations of the $\mathrm{D} / \mathrm{H}$ ratios in disks enable the comparison of the fractionation measured in comets such as Hale-Bopp (Blake et al. 1999) with the specific conditions at each disk radius. We have presented the first images of $\mathrm{DCO}^{+}$ and DCN emission from the disk around a classical T Tauri star, TW Hydrae, along with images of the $\mathrm{HCN}$ and $\mathrm{HCO}^{+} J=3-2$ lines. The observations of deuterium fractionation serve as a clear measure of the importance of low-temperature gas-phase deuterium fractionation processes. These observations strongly support 

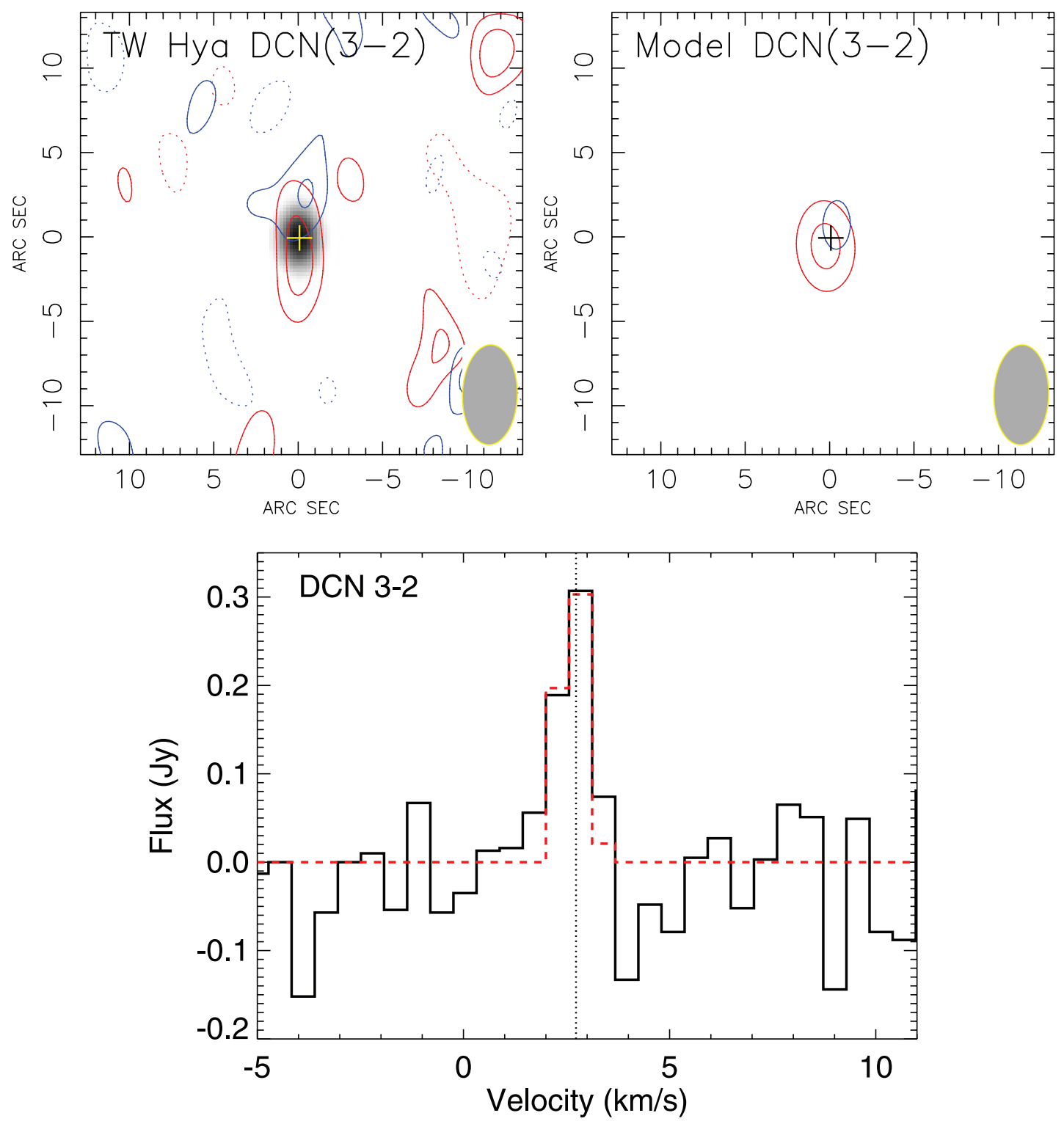

FIG. 12.-Top left: DCN $J=3-2$ velocity channel maps (red contours, $2.84 \mathrm{~km} \mathrm{~s}^{-1}$; blue contours, $2.28 \mathrm{~km} \mathrm{~s}^{-1}$ ) from TW Hydrae, overlaid on the 217 GHz dust continuum map (gray scale). The cross indicates the position of the continuum peak. Top right: Simulated model for DCN $J=3-2$. The $1 \sigma$ contour step is 0.09 Jy beam ${ }^{-1}$, and the contours start with $2 \sigma$. Bottom: Beam-averaged DCN 3-2 spectra at the continuum (stellar) position. The SMA data are indicated by the solid histogram and the simulated model by the dashed histogram. The vertical dotted line indicates the position of the fitted $V_{\mathrm{LSR}}$ for $\operatorname{HCN} J=3-2$.

the proposed link among high gas densities, cold temperatures, and enhanced deuterium fractionation. Detailed chemical models are still needed to explain how $\mathrm{DCO}^{+}$disappears from the outer part of the disk.

The similarity of the $\mathrm{D} / \mathrm{H}$ ratios in cold clouds, disks, and pristine cometary material has been used to argue that the gas spends most of its lifetime at low temperatures and is incorporated into the disks before the envelope is heated, i.e., before the class I stage. By combining self-consistent physical models and twodimensional radiative transfer codes to interpret high spatial resolution millimeter-wave molecular images, we are only now beginning to investigate the radial and vertical distributions of molecules in disks. The radial distribution of $\mathrm{DCO}^{+}$in the disk of TW Hydrae indicates that in situ deuterium fractionation is ongoing. The molecular evolution within disks must therefore be considered in the investigation of the origin of primitive solar system bodies.
We have obtained less stringent constraints on the vertical distributions of molecules in the disk of TW Hydrae. To address the ambiguity present in the analysis of single objects, data from a robust sample of disks are needed, in particular one that covers a range of disk inclinations. More sensitive observations are also needed for the rare isotopologues $\mathrm{H}^{13} \mathrm{CN}, \mathrm{H}^{13} \mathrm{CO}^{+}$, and, of course, $\mathrm{DCN}$, to understand the radial and vertical gradient of deuterated species in these disks. In the future, observations of DCN and other species with the Atacama Large Millimeter Array will provide further insight into the chemical state of protoplanetary disks.

Partial support for this work comes from NASA Origins of Solar Systems Grant NNG05GI81G. M. R. H. is supported by a VIDI grant from the Netherlands Organization for Scientific Research. C. Q. acknowledges Paola Caselli for her help and useful suggestions. We thank the referee for very useful comments. 


\section{REFERENCES}

Aikawa, Y., \& Herbst, E. 1999, ApJ, 526, 314

Aikawa, Y., Miyama, S. M., Nakano, T., \& Umebayashi, T. 1996, ApJ, 467, 684

Aikawa, Y., \& Nomura, H. 2006, ApJ, 642, 1152

Aikawa, Y., van Zadelhoff, G. J., van Dishoeck, E. F., \& Herbst, E. 2002, A\&A, 386,622

Beckwith, S. V. W., \& Sargent, A. I. 1993, ApJ, 402, 280

Bergin, E. A., Aikawa, Y., Blake, G. A., \& van Dishoeck, E. F. 2007, in Protostars and Planets V, ed. B. Reipurth, D. Jewitt, \& K. Keil (Tucson: Univ. Arizona Press), 751

Blake, G. A., Qi, C., Hogerheijde, M. R., Gurwell, M. A., \& Muhleman, D. O. 1999, Nature, 398, 213

Calvet, N., D’Alessio, P., Hartmann, L., Wilner, D., Walsh, A., \& Sitko, M. 2002, ApJ, 568, 1008

Caselli, P. 2002, Planet. Space Sci., 50, 1133

Caselli, P., van der Tak, F. F. S., Ceccarelli, C., \& Bacmann, A. 2003, A\&A, 403, L37

Ceccarelli, C., \& Dominik, C. 2005, A\&A, 440, 583

Ceccarelli, C., Dominik, C., Caux, E., Lefloch, B., \& Caselli, P. 2005, ApJ, 631, L81

Ceccarelli, C., Dominik, C., Lefloch, B., Caselli, P., \& Caux, E. 2004, ApJ, 607, L51
Dutrey, A., Guilloteau, S., \& Ho, P. 2007, in Protostars and Planets V, ed. B. Reipurth, D. Jewitt, \& K. Keil (Tucson: Univ. Arizona Press), 495 Flower, D. R. 1999, MNRAS, 305, 651

Green, S., \& Thaddeus, P. 1974, ApJ, 191, 653

Guilloteau, S., Piétu, V., Dutrey, A., \& Guélin, M. 2006, A\&A, 448, L5

Ho, P. T. P., Moran, J. M., \& Lo, K. Y. 2004, ApJ, 616, L1

Hogerheijde, M. R., \& van der Tak, F. F. S. 2000, A\&A, 362, 697

Isella, A., Testi, L., Natta, A., Neri, R., Wilner, D., \& Qi, C. 2007, A\&A, 469, 213

Meier, R., et al. 1998, Science, 279, 1707

Qi, C., Wilner, D. J., Calvet, N., Bourke, T. L., Blake, G. A., Hogerheijde, M. R., Ho, P. T. P., \& Bergin, E. 2006, ApJ, 636, L157

Qi, C., et al. 2004, ApJ, 616, L11

Schöier, F. L., van der Tak, F. F. S., van Dishoeck, E. F., \& Black, J. H. 2005 , A\&A, 432, 369

Setiawan, J., Henning, T., Launhardt, R., Müller, A., Weise, P., \& M., K. 2008, Nature, 451, 38

Stark, R., van der Tak, F. F. S., \& van Dishoeck, E. F. 1999, ApJ, 521, L67

Thi, W.-F., van Zadelhoff, G.-J., \& van Dishoeck, E. F. 2004, A\&A, 425, 955 van Dishoeck, E. F., Thi, W.-F., \& van Zadelhoff, G.-J. 2003, A\&A, 400, L1

Vastel, C., Phillips, T. G., \& Yoshida, H. 2004, ApJ, 606, L127

Willacy, K. 2007, ApJ, 660, 441 